\title{
Hierarchical potential differentiation of liver cancer stem cells
}

\author{
Wenfeng Zhang ${ }^{1, B-E}, D_{i} M^{2, B-E}$, Kai Feng ${ }^{3, A, F}$ \\ ${ }^{1}$ Chongqing Key Laboratory of Hepatobiliary Surgery and Department of Hepatobiliary Surgery, Second Affiliated Hospital of Chongqing Medical University, Chongqing, China \\ ${ }^{2}$ Key Laboratory of Molecular Biology for Infectious Diseases of the Ministry of Education of China, Second Affiliated Hospital of Chongqing Medical University, Chongqing, China \\ ${ }^{3}$ Institute of Hepatobiliary Surgery, Southwest Hospital, $3^{\text {rd }}$ Military Medical University, Chongquing, China \\ A - research concept and design; $\mathrm{B}$ - collection and/or assembly of data; $\mathrm{C}$ - data analysis and interpretation; \\ $D$ - writing the article; $E$ - critical revision of the article; $F$ - final approval of article
}

\section{Address for correspondence \\ Kai Feng \\ E-mail: fengkai7688@hotmail.com}

\section{Funding sources}

China Postdoctoral Science Foundation funded project (XM20120013) and Fund of State Key Laboratory of

Trauma, Burns and Combined Injury (SKLKF201209).

\section{Conflict of interest}

None declared

\section{Acknowledgements}

This review was performed by the Key Laboratory of Trauma, Burns and Combined Injury, Chongqing Engineering Research Center for Nanomedicine, College of Preventive Medicine, Third Military Medical University and the Chongqing Key Laboratory of Hepatobiliary Surgery and the Department of Hepatobiliary Surgery, Second Affiliated Hospital of Chongqing Medical University.

\begin{abstract}
Hepatocellular carcinoma (HCC) is one of the most malignant tumors in Chinese people and offers poor prognosis. Tumor tissue, like normal tissue, is hierarchically differentiated. Thus, minor tumor cell populations able to differentiate, such as stem cells, sustain tumor self-renewal and proliferation. The fact that liver cancer stem cells (CSCS) with different surface markers appear heterogeneous with respect to oncogenesis and drug resistance indicates that subpopulations of surface markers preserve the hierarchical potential of differentiation during proliferation, deterioration and relapse. The epithelial to mesenchymal transition (EMT) is correlated to tumor malignancy and aggression, and hepatocytes bearing EMT have obvious hierarchical differentiation potential with respect to signaling pathways such as transforming growth factor $\beta$, Wnt/B-catenin and microRNA. Therefore, it may be more effective for early diagnosis to monitor $\mathrm{HCC}$ recurrence using peripherally circulating $\mathrm{CSC}$, and these may also offer potential for HCC immunotherapy or for targeting HCC treatment using these markers. Thus, we reviewed the generation, hierarchical differentiation and clinical application of hepatic CSCs.
\end{abstract}

Key words: tumor heterogeneity, tumorigenesis, liver cancer stem cells, surface molecular markers

DOI

10.17219/acem/66343

\section{Copyright}

Copyright by Author(s)

This is an article distributed under the terms of the

Creative Commons Attribution Non-Commercial License

(http://creativecommons.org/licenses/by-nc-nd/4.0/) 


\section{Introduction}

A high-grade malignant solid tumor with dismal prognoses, hepatocellular carcinoma (HCC) represents the $4^{\text {th }}$ most common malignant neoplasm in Chinese people. ${ }^{1}$ Although studies of $\mathrm{HCC}$ are advancing, methods for early and effective diagnosis of $\mathrm{HCC}$ require improvement. ${ }^{2-3}$ Once HCC symptoms appear, the cancer is often very advanced and includes intrahepatic or systemic metastasis. ${ }^{4}$ Poorly timed identification, late treatment and postoperative relapse contribute to high HCC mortality. ${ }^{5}$ Although much effort to understand $\mathrm{HCC}$ recurrence has been put forth, the causes of therapeutic resistance are unclear. Cancer stem cells (CSCs), which may also be considered tumor initiating cells (TICs), may be the source from which HCC arises. In addition, CSCs may be the chief cause of tumor heterogeneity, which may contribute to drug resistance. ${ }^{6-9}$ The hierarchical differentiation of hepatic CSCs and immunologic escape of self-monitoring are connected to tumorigenic stage. ${ }^{6}$ Meanwhile, signaling pathways and genes that regulate stem cell differentiation may contribute to the control and maintenance of CSC differentiation, such as transforming growth factor $\beta$ (TGF- $\beta$ ), Wnt $/ \beta$-catenin and microRNA. ${ }^{8-9}$ Thus, to better understand the etiology of HCC and address drug resistance, we must understand liver CSCs.

\section{Research of the hierarchical differentiation potential of CSCs}

Neoplasms are thought to represent the clonal proliferation of malignant tissue driven by genomic and genetic instabilities that are unrelated to mutation, selection or adaptation. ${ }^{6}$ Recent data points to the importance of identification of genotypes among tumor patients because interpatient heterogeneity may drive treatment advances in oncology and may guide individualized therapies. ${ }^{7}$ However, different genetic subclones have been identified in the same tumor and within the same pathological tumor classification with different genetic phenotypes. Then, over time, these subgroup clones behave differently, or cause tumor or intratumor heterogeneity, which are thought to be critical for tumorigenesis and metastasis. ${ }^{8,9}$

The generation of heterogeneity is related to the differentiation potential of embryonic stem cells that develop into various functional organs. ${ }^{9}$ Thus, $\mathrm{HCC}$ is a stem cell abnormality and their ability to self-renew and differentiate is one of their most critical features, hence their reclassification as CSCs or TICs. These cells can initiate tumor formation after administration into nude mice, and they subsequently differentiate to different functional cancer cells in the lesions from which they are derived.

CSCs have 2 hypothetical contributions to oncogenesis and tumor heterogeneity. First, every tumor cell in the same tumor lesion is created with a potential for differ- entiation and a specific tumor cell population will proliferate and differentiate to stimulate tumor growth. ${ }^{7}$ Next, the tumor lesion is hierarchically organized as is normal tissue differentiation from embryonic stem cells. Few CSCs can proliferate and sustain long-term tumor growth and the remaining tumor cells are silent due to a silencing of differentiation potential by the hierarchical organization. ${ }^{7}$ Strictly speaking, CSCs have the same genotype as other tumor cells in the same tumor lesion, and are only unique after post-translational modifications and epigenetic events. Investigators have reported that this may be due to associations with the mechanism of normal tissue differentiation from embryonic stem cells. ${ }^{10}$ All cancer cells originate from one precursor (the CSC) and in a tumor microenvironment, HCC progenitor cells acquire autocrine IL- 6 signaling to stimulate growth and malignant progression for CSCs via upregulation of LIN28 expression. ${ }^{11}$ Although all types of hepatic lineages can acquire oncogenic reprogramming to CSCs, how this occurs and why requires more study. ${ }^{12-13}$

\section{Obtaining hepatic CSCs}

The hepatic CSC theory has been promoted since 1989, and it suggests that a few specific hepatic cancer cell lesions obtain differentiation potential and cause malignant differentiation of HCCs by altering enzyme structures. ${ }^{10}$ However, limited technologies at that time prevented direct data to support the existence of liver CSCs. Now, with immunofluorescence, immunomagnetic beads and flow cytometry, we can procure liver CSCs using liver cancer cell markers. ${ }^{14}$ These methods also support research into mechanism of HCC heterogeneity and various hepatic CSC surface markers have been confirmed. At present, 10 hepatic CSC markers such as CD133, CD90, and CD44 have been reported. ${ }^{15}$

CD133, or Prominin-1, is highly expressed in fetal liver progenitor cells and is a member of the hematopoietic stem/progenitor cell transmembrane glycoproteins. CD133 is the earliest and most widely used protein for the separation of liver CSC subpopulations. ${ }^{16-18}$ Stephanie reported that CD133-positive liver cancer cells were more tumorigenic than CD133-negative liver cancer cells in vivo and in vitro. ${ }^{19}$ Also, stem cell-related genes such as Notch, $\beta$-catenin and Oct $3 / 4$ are highly expressed in the CD133-positive subpopulation. ${ }^{20}$

CD90, or Thy-1, is a surface marker of hepatic oval cells and CD45-negative CD90-positive subpopulations exists in hepatic tumor lesions and in the peripheral circulation. ${ }^{20}$ Yang's group identified CD90-positive CD44positive and CD45-negative markers for CSCs from liver lesions and the peripheral circulation, but they report that these stem cells have increased tumorigenicity and invasiveness compared to CD133-positive subpopulations. ${ }^{21-23}$ 
CD44 is a cell surface adhesion molecule that binds to extracellular ligand to regulate hepatic cancer proliferation, differentiation, metastasis and survival. ${ }^{16,24-25}$ Moreover, CD45-positive subpopulations often express other stem cell markers such as CD133 and CD90. ${ }^{21,26}$ Yang and co-workers suggest that CD44-positive liver CSC subpopulations are more aggressive than CD44negative subpopulations. ${ }^{21}$ Furthermore, Zhu's group reported that CD44-positive CD133-positive liver CSCs not only excessively express stem cell-related genes, but are more resistant to chemotherapy than CD44-negative CD133-positive cells. ${ }^{26}$

High expression of CD13, which is regarded to be a marker for liver cancer that is dormant or half dormant, occurs during the G1/G0 phase. ${ }^{27-29}$ Haraguchi's group reported that CD13-positive hepatic cancer cells are more resistant to 5-FU and Adriamycin and have greater oncogenicity. ${ }^{29}$ Also, CD13 expression was excessively induced by 5 -FU and Adriamycin in liver cancer cells. ${ }^{29}$

Epithelial cell adhesion molecule (EpCAM), or CD326, is highly expressed in embryonic liver cells, bile duct epithelial cells, and hyperplastic bile duct epithelial cells in liver cirrhosis. ${ }^{30}$ Yamashita et. al. reported that EpCAM is expressed more in liver cancer and the molecular signaling pathways of EpCAM-positive liver cancer cells are closely related to liver progenitor cells. ${ }^{31-33}$ Simultaneously, Terris' group reported that EpCAM-positive AFPpositive liver cancer cells are more tumorigenic, mobile and invasive to the portal vein. ${ }^{31}$ Therefore, EpCAM is thought to be a marker of liver CSCs.

OV6 is a marker of hepatic oval cells and is closely related to hepatic disease. ${ }^{34,35}$ OV6-positive liver cancer cells are said to have more resistance to chemotherapy in vitro ${ }^{36}$ and they are more aggressive in patients as well as are closely related to the clinical pathological characteristics of liver cancer. ${ }^{37}$ However, OV6-positive patients with liver cancer are not unusually different with respect to disease-free and overall survival compared to OV6negative patients with liver cancer. ${ }^{37}$

SALL4, a member of the zinc finger transcription factor family, is a stem cell marker. ${ }^{38,39}$ Oikawa's group suggested that sal-like protein (SALL4) provokes the proliferation of liver cancer cells in vitro and induces overexpression of various liver CSC surface markers such as EpCAM, ATPbinding cassette-G2 (ABCG2) and CK19. ${ }^{39}$ Others regard SALL4 to be a marker of liver CSCs, which can be used to predict HCC treatment outcomes. ${ }^{40-42}$ Furthermore, expression of phosphatase and tensin homologue protein was suppressed and the formation of tumors in xenograft models was inhibited by overexpression of SALL4. ${ }^{43}$ Thus, inhibiting expression of SALL4 can inhibit proliferation and differentiation of liver cancer cells. ${ }^{44-48}$

Many laboratories have reported that different liver cancer subpopulations sorted by surface markers have diverse features. For example, Zen and colleagues reported that liver cancer subpopulations sorted by ABCG2 have drug tolerance ${ }^{49}$ More interestingly, aldehyde dehydrogenase $(A L D H)$ is not a stem cell surface marker but ALDHpositive CD133-positive cells have greater tumorigenicity and invasiveness. ${ }^{50}$ Recently, toll-like receptors 4 and interleukin-6 were referred to as liver CSC markers due to their relationship with inflammation and tumorigenesis. ${ }^{11,51}$ However, these subpopulations marked by different surface proteins maintain orderly differentiation potential during proliferation, deterioration and relapse in liver cancer and these may be the source of cancer heterogeneity. Thus, investigations of the mechanisms of liver CSC surface markers during cancer proliferation, metastasis and relapse may allow the development of agents that can inhibit tumor growth by targeting subpopulations of CSCs with unique differentiation potentials.

\section{Mechanism of tumor heterogeneity produced by liver CSCs}

Epithelial-to-mesenchymal transition (EMT) is closely related with the degree of liver cancer malignance and aggression, and liver cancer cells bearing EMT have obvious hierarchical differentiation potential closely associated with signaling pathways such as transforming growth factor $\beta$ (TGF- $\beta$ ), Wnt/ $\beta$-catenin and microRNA. ${ }^{37,52,53}$

Knock-out of TGF- $\beta$ inhibits the incidence of liver cancer in nude mice and overexpression of TGF- $\beta$ not only increases the oncogenicity of liver cancer via highly expressed transcription factors related to EMT in different tumor cells such as TWIST1, TWIST2, SNAI1, SNAI2, ZEB1 and ZEB2, but also makes the cells more likely to metastasize. ${ }^{54}$ Also, E-cadherin is inhibited by overexpression of TGF- $\beta$ and high expression of TWIST1 has been discovered in CD44-positive CD24-negative breast CSCs with more obvious potential for differentiation and more aggressive behavior. ${ }^{55,56}$ In addition, TGF- $\beta$ signaling alters the patterns of liver tumorigenesis induced by PTEN inactivation in liver CD133-positive CSCs but how tumor heterogeneity is regulated by TWIST1 is unclear.

Leal and colleagues reported that microRNA 181 (miR181 ) is highly expressed in embryonic liver and EpCAMpositive AFP-positive liver cancer subpopulations. ${ }^{57,58}$ Also, the tumorigenicity of liver CSCs is significantly suppressed by inhibition of miR-181 which chiefly functions to regulate the differentiation potential of liver CSCs by activating transcription factors such as caudal homeobox gene 2 (CDX2), transcription factor-GATA6 and by negatively regulating the $\mathrm{Wnt} / \beta$-catenin pathway via nemo-like kinase (NLK). ${ }^{52,57,58}$ Moreover, let-7 and Lin28 have been reported to be related to the growth and metastasis of HCC. Lin28 is highly expressed in normal embryonic stem cells and maintains the self-renewal of liver CSCs by inhibiting the combination of let-7 with mature microRNA. ${ }^{59}$ Research into microRNA suggests that positive and negative pathways exist for regulating 
the differentiation potential of liver CSCs via different microRNAs. ${ }^{58,59}$ For negative regulation, degraded let7 by excessively active Lin 28 and c-MYC disequilibrate liver CSCs and all cancer cells that accelerate the growth and metastasis of HCC. ${ }^{59}$ Meanwhile, with positive regulation, high expression of EpCAM, which is regarded as a prominent marker of liver CSCs, is mediated by inhibition of TGF- $\beta$ by downstream transcription factors of miR-18 such as CDX2, GATA6 and NLK. ${ }^{53,54}$ EpCAM intracellular domain (EpICD), which is a lysate from EpCAM, enters the nucleus and induces overexpression of cyclin D1, c-MYC and miR-181 after combining with LIM domain protein 2 (FHL2), $\beta$-catenin and lymphoid enhancer factor 1 (Lef-1). ${ }^{58}$ In addition, Liu's group asserted that TGF- $\beta 1$ acts through miR-155 to down-regulate TP53INP1 to promote EMT and liver CSC phenotypes. ${ }^{60}$ The Wnt/ $\beta$-catenin signaling pathway that regulates tumor heterogeneity is mainly related to microRNA but how this occurs to sustain the balance of liver CSCs and cancer cells has not been elucidated.

\section{Function of liver CSCs for diagnosis and treatment of HCC}

Evidence is accumulating that CSCs are a source of cancer cell and tumor heterogeneity. ${ }^{6-9}$ Yang's group reported that liver CSCs from the peripheral circulation had greater tumorigenicity and confirmed that these were significantly positively related to a 2-year relapse after resection. ${ }^{23}$ Various liver CSC-related proteins have been reported to be associated with malignance of liver cancer, such as K19 and c-kit. ${ }^{61} \mathrm{~K} 19$ is a keratin on the bile duct cell and c-kit, or CD117, is a stem cell factor receptor. K19 and c-kit are reported to be associated with a range of vascular invasion, tumor diameters, degree of cancer differentiation and APF. ${ }^{61}$ Obtaining liver CSCs from the peripheral blood has been made easier with flow cytometry and immunomagnetic beads. ${ }^{14,19}$

Liu's group reported that CD90 has a specificity of 91.9\% for HCC and a sensitivity of $48.22 \%$ for predicting poor differentiation. ${ }^{62}$ In addition, TLR4-positive CSCs were found to be significantly greater in HCC tissues with microvascular invasion and strongly associated with both early recurrence and poor patient survival. ${ }^{51}$ Thus, high expression of these markers is significantly associated with poor response to treatment and reduced survival, and measuring and/or monitoring liver CSCs in liver lesions or the peripheral circulation may be an effective tool for surveilling growth, metastasis and relapse of HCC.

Stem cell heterogeneity indicates tolerance for cell death induced by DNA damage due to a survival advantage from their full potential of gene expression ${ }^{6-9}$ and this was discovered in liver CSCs that are more tolerant to chemotherapy and radiotherapy and have greater relapse and metastasis after diverse interventions com- pared to quiescent cancer cells. It has been reported that a specific antibody targeted to EpCAM can more effectively inhibit liver cancer growth that conventional chemotherapy. ${ }^{31-33,59}$ In addition, liver CSCs influence sorafenib resistance via Akt/pi3k, WNT, Notch, and IL-6 pathways. ${ }^{63}$ Therefore, specific inhibitors targeted to surface markers that directly kill liver CSCs or restrain selfrenewal and differentiation may hold promise for tumor treatment. ${ }^{64-66}$

\section{Conclusions}

Higher oncogenicity has been reported for hepatic CSC surface markers to suggest a source of tumor heterogeneity but at what stage of differentiation these markers are best monitored is uncertain. Additionally, various liver CSC subpopulations may be more tolerant to radiotherapy or chemotherapy and this may explain the tumor heterogeneity due to hepatic CSC post-translational modifications and epigenetic differences of hierarchical differentiation. Current evidence suggests that measuring peripheral circulating CD90-positive liver CSCs may be useful for early diagnosis and for monitoring potential tumor relapse. Meanwhile, antibodies targeted to liver CSCs with IL-6 or its inhibitors to inhibit self-renewal and survival signaling pathways may hold promise for immunotherapy.

\section{References}

1. Chen W, Zheng R, Zeng H, Zhang S, He J. Annual report on status of cancer in China, 2011. Chin J Cancer Res. 2015;27:2-12.

2. Farrell GC, Chan HL, Yuen MF, et al. Prevention of hepatocellular carcinoma in the Asia-Pacific region: Consensus statements. J Gastroenterol Hepatol. 2010;25:657-663.

3. Amarapurkar D, Han KH, Chan HL, Ueno Y. Application of surveillance programs for hepatocellular carcinoma in the Asia-Pacific Region. J Gastroenterol Hepatol. 2009;24:955-961.

4. Llovet JM, Bruix J. Novel advancements in the management of hepatocellular carcinoma in 2008. J Hepatol. 2008;48:S20-37.

5. Llovet JM, Fuster J, Bruix J. The Barcelona approach: Diagnosis, staging, and treatment of hepatocellular carcinoma. Liver Transpl. 2004;10:S115-120.

6. Klein CA. Selection and adaptation during metastatic cancer progression. Nature. 2013;501:365-372.

7. Beck B, Blanpain C. Unravelling cancer stem cell potential. Nat Rev Cancer. 2013;13:727-738.

8. Bedard PL, Hansen AR, Ratain MJ, Siu LL. Tumour heterogeneity in the clinic. Nature. 2013;501:355-364.

9. Meacham CE, Morrison SJ. Tumour heterogeneity and cancer cell plasticity. Nature. 2013;501:328-337.

10. Valent $P$, Bonnet $D$, De Maria $R$, et al. Cancer stem cell definitions and terminology: The devil is in the details. Nat Rev Cancer. 2012;12:767-775.

11. He G, Dhar D, Nakagawa $H$, et al. Identification of liver cancer progenitors whose malignant progression depends on autocrine IL-6 signaling. Cell. 2013;155:384-396.

12. Holczbauer A, Factor VM, Andersen JB, et al. Modeling pathogenesis of primary liver cancer in lineage-specific mouse cell types. Gastroenterology. 2013;145:221-231.

13. Li J, Yu Y, Wang J, et al. Establishment of a novel system for the culture and expansion of hepatic stem-like cancer cells. Cancer Lett. 2015;360:177-186. 
14. Zhang Y, Li J, Cao L, Xu W, Yin Z. Circulating tumor cells in hepatocellular carcinoma: detection techniques, clinical implications, and future perspectives. Semin Oncol. 2012;39:449-460.

15. Tong CM, Ma S, Guan XY. Biology of hepatic cancer stem cells. J Gastroenterol Hepatol. 2011;26:1229-2337.

16. Turner R, Lozoya O, Wang Y, et al. Human hepatic stem cell and maturational liver lineage biology. Hepatology. 2011;53:1035-1045.

17. Mizrak D, Brittan M, Alison M. CD133: Molecule of the moment. J Pathol. 2008;214:3-9.

18. Miraglia S, Godfrey W, Yin AH et al. A novel five-transmembrane hematopoietic stem cell antigen: Isolation, characterization, and molecular cloning. Blood. 1997;90:5013-5021.

19. Ma S. Biology and clinical implications of CD133(+) liver cancer stem cells. Exp Cell Res. 2013;319:126-132.

20. Dennis JE, Esterly K, Awadallah A, Parrish CR, Poynter GM, Goltry KL. Clinical-scale expansion of a mixed population of bone-marrowderived stem and progenitor cells for potential use in bone-tissue regeneration. Stem Cells. 2007;25:2575-2582.

21. Fan ST, Yang ZF, Ho DW, Ng MN, Yu WC, Wong J. Prediction of pos thepatectomy recurrence of hepatocellular carcinoma by circulating cancer stem cells: A prospective study. Ann Surg. 2011;254:569-576.

22. Yang ZF1, Ho DW, Ng MN, et al. Significance of CD90+ cancer stem cells in human liver cancer. Cancer Cell. 2008;13:153-166.

23. Yang ZF, Ngai P, Ho DW, et al. Identification of local and circulating cancer stem cells in human liver cancer. Hepatology. 2008:471:919-928.

24. Calloni R, Cordero EA, Henriques JA, Bonatto D. Reviewing and updating the major molecular markers for stem cells. Stem Cells Dev. 2013;22,1455-1476.

25. Nagano O, Okazaki S, Saya H. Redox regulation in stem-like cancer cells by CD44 variant isoforms. Oncogene. 2013;32:5191-5198.

26. Zhu Z, Hao X, Yan M, et al. Cancer stem/progenitor cells are highly enriched in CD133+CD44+ population in hepatocellular carcinoma. Int J Cancer. 2010;126:2067-2078.

27. Christ B, Stock $P$, Dollinger MM. CD13: Waving the flag for a nove cancer stem cell target. Hepatology. 2011;53:1388-1390.

28. Marusawa $\mathrm{H}, \mathrm{Chiba} \mathrm{T}$. A marker for dormant cancer stem cells in human hepatocellular carcinoma. Gastroenterology. 2011;140:1353-1355.

29. Haraguchi N, Ishii H, Mimori K, et al. CD13 is a therapeutic target in human liver cancer stem cells. J Clin Invest. 2010;120:3326-3339.

30. Kim JW, Ye Q, Forgues M, et al. Cancer-associated molecular signature in the tissue samples of patients with cirrhosis. Hepatology. 2004;39:518-527.

31. Terris $B$, Cavard C, Perret C. EpCAM, a new marker for cancer stem cells in hepatocellular carcinoma. J Hepatol. 2010;52:280-281.

32. Yamashita T, Ji J, Budhu A, et al. EpCAM-positive hepatocellular carcinoma cells are tumor-initiating cells with stem/progenitor cell features. Gastroenterology. 2009;136:1012-1024.

33. Yamashita T, Forgues $M$, Wang W, et al. EpCAM and alpha-fetoprotein expression defines novel prognostic subtypes of hepatocellular carcinoma. Cancer Res. 2008;68:1451-1461.

34. Strain AJ, Crosby HA, Nijjar S, Kelly DA, Hubscher SG. Human liverderived stem cells. Semin Liver Dis. 2003;23:373-384.

35. Roskams T, De Vos R, Van Eyken P, Myazaki H, Van Damme B, Des met V. Hepatic OV-6 expression in human liver disease and rat experiments: Evidence for hepatic progenitor cells in man. $J$ Hepatol. 1998;29:455-463.

36. Yang W, Wang C, Lin Y, et al. OV6(+) tumor-initiating cells contribute to tumor progression and invasion in human hepatocellular carcinoma. J Hepatol. 2012;57:613-620.

37. Yang W, Yan HX, Chen L, et al. Wnt/beta-catenin signaling contributes to activation of normal and tumorigenic liver progenitor cells. Cancer Res. 2008;68:4287-4295.

38. Fagoonee S, Hobbs RM, De Chiara L, et al. Generation of functional hepatocytes from mouse germ line cell-derived pluripotent stem cells in vitro. Stem Cells Dev. 2010;19:1183-1194.

39. Oikawa T, Kamiya A, Kakinuma $S$, et al. Sall4 regulates cell fate decision in fetal hepatic stem/progenitor cells. Gastroenterology. 2009;136:1000-1011.

40. Zeng SS, Yamashita $T$, Kondo $M$, et al. The transcription factor SALL4 regulates stemness of EpCAM-positive hepatocellular carcinoma. J Hepatol. 2014;60:127-134.

41. Oikawa T, Kamiya A, Zeniya M, et al. Sal-like protein 4 (SALL4), a stem cell biomarker in liver cancers. Hepatology. 2013;57:1469-1483.
42. Marquardt JU, Thorgeirsson SS. Sall4 in "stemness"-driven hepatocarcinogenesis. N Engl J Med. 2013;368:2316-2318.

43. Yong KJ, Gao C, Lim JS, et al. Oncofetal gene SALL4 in aggressive hepatocellular carcinoma. N Engl J Med. 2013;368:2266-2276.

44. Yong KJ, Chai L, Tenen DG. Oncofetal gene SALL4 in aggressive hepatocellular carcinoma. N Engl J Med. 2013;369:1171-1172.

45. Masuda S, Suzuki K, Izpisua BJC. Oncofetal gene SALL4 in aggressive hepatocellular carcinoma. N Engl J Med. 2013;369:1171.

46. Suzuki E, Chiba T, Yokosuka O. Oncofetal gene SALL4 in aggressive hepatocellular carcinoma. N Engl J Med. 2013, 369, 1170-1171.

47. Hopkins LJ, Rowe IA, Houlihan DD: Oncofetal gene SALL4 in aggressive hepatocellular carcinoma. N Engl J Med. 2013;369:1170.

48. Leake I. Cancer: Importance of oncofetal gene, SALL4, in a subset of hepatocellular carcinoma. Nat Rev Gastroenterol Hepatol. 2013;10:441

49. Zen Y, Fujii T, Yoshikawa S, et al. Histological and culture studies with respect to ABCG2 expression support the existence of a cancer cell hierarchy in human hepatocellular carcinoma. Am J Pathol. 2007:170:1750-1762.

50. Ma S, Chan KW, Lee TK, et al. Aldehyde dehydrogenase discriminates the CD133 liver cancer stem cell populations. Mol Cancer Res. 2008;6:1146-1153.

51. Liu WT, Jing YY, Yu GF, et al. Toll like receptor 4 facilitates invasion and migration as a cancer stem cell marker in hepatocellular carcinoma. Cancer Lett. 2015;358:136-143.

52. Bessède E, Staedel C, Acuña Amador LA, et al. Helicobacter pylori generates cells with cancer stem cell properties via epithelial-mesenchymal transition-like changes. Oncogene. 2014;33:4123-4131.

53. Acloque H, Adams MS, Fishwick K, Bronner-Fraser M, Nieto MA. Epithelial-mesenchymal transitions: The importance of changing cell state in development and disease. J Clin Invest. 2009;119:1438-1449.

54. Thiery JP, Acloque H, Huang RY, Nieto MA. Epithelial-mesenchymal transitions in development and disease. Cell. 2009;139:871-890.

55. Majumdar A, Curley SA, Wu X, et al. Hepatic stem cells and transforming growth factor beta in hepatocellular carcinoma. Nat Rev Gastroenterol Hepatol. 2012;9:530-528.

56. Al-Hajj M, Wicha MS, Benito-Hernandez A, Morrison SJ, Clarke MF. Prospective identification of tumorigenic breast cancer cells. Proc Natl Acad Sci U S A. 2003;100:3983-398.

57. Leal JA, Lleonart ME. MicroRNAs and cancer stem cells: Therapeutic approaches and future perspectives. Cancer Lett. 2013;338:174-183.

58. Ji J, Yamashita T, Budhu A, et al. Identification of microRNA-181 by genome-wide screening as a critical player in EpCAM-positive hepatic cancer stem cells. Hepatology. 2009;50:472-480.

59. Heo I, Joo C, Cho J, Ha M, Han J, Kim VN. Lin28 mediates the terminal uridylation of let-7 precursor MicroRNA. Mol Cell. 2008;32:276-284.

60. Liu F, Kong X, Lv L, Gao J. TGF-beta1 acts through miR-155 to downregulate TP53INP1 in promoting epithelial-mesenchymal transition and cancer stem cell phenotypes. Cancer Lett. 2015;359:288-298.

61. Kim H, Choi GH, Na DC, et al. Human hepatocellular carcinomas with "Stemness"-related marker expression: Keratin 19 expression and a poor prognosis. Hepatology. 2011;54:1707-1717.

62. Liu R, Shen Y, Nan K, et al. Association between expression of cancer stem cell markers and poor differentiation of hepatocellular carcinoma: A meta-analysis (PRISMA). Medicine. 2015;94:e1306.

63. Chen J, Jin R, Zhao J, et al. Potential molecular, cellular and microenvironmental mechanism of sorafenib resistance in hepatocellular carcinoma. Cancer Lett. 2015;367:1-11.

64. You N, Zheng L, Liu W, Zhong X, Wang W, Li J. Proliferation inhibition and differentiation induction of hepatic cancer stem cells by knockdown of BC047440: A potential therapeutic target of stem cell treatment for hepatocellular carcinoma. Oncol Rep. 2014:31:1911-1920.

65. Rountree $C B$, Mishra L, Willenbring $H$. Stem cells in liver diseases and cancer: Recent advances on the path to new therapies. Hepatology. 2012;55:298-306.

66. Pang RW, Poon RT. Cancer stem cell as a potential therapeutic target in hepatocellular carcinoma. Curr Cancer Drug Targets. 2012;12:1081-1094. 